\title{
Unified Modeling and Measurement of Current-Programmed Converters
}

\author{
F. Dong Tan and R. D. Middlebrook \\ Power Electronics Group, 116-81, Caltech, Pasadena, CA 91125
}

Tel: (818)356-4835, Fax: (818)356-2944

\begin{abstract}
A unified model is established for a current-programmed converter, which is both a modification and an extension of familiar models. Inclusion of the sampling effect allows the presence of an additional pole in the currentloop gain to be inferred. The resulting final double-slope asymptote is fixed in position, and the crossover frequency cannot exceed half the switching frequency. A new "stability parameter" $Q$ s determines the additional pole and describes the degree of peaking in the closed-loop transfer functions. Experimental verification employs an analog signal injection technique.
\end{abstract}

\section{Introduction}

Current programming has become the regulating scheme of choice in dc-to-dc converters owing to its advantages over duty-ratio programming such as better line-noise rejection, automatic overload protection, easy paralleling of multiple converters, and especially design flexibility in improving small-signal dynamics.

A large number of small-signal models have been proposed, for example, [1-9]. A low-frequency circuitoriented approach was proposed in [4] in which a dutyratio control law is developed that is consistent with the state-space averaged model for the power stage. This approach has gained acceptance owing to its simplicity and the insight gained into the properties of current programming.

The duty-ratio control law in [4] was derived by perturbation of an expression for inductor average current in steady state. This procedure was disputed in [5], where it is argued that the small-signal duty-ratio control law needs to be derived by perturbing an expression for inductor average current in perturbed state. Experimental measurements, however, did not support its

This work was conducted under the Power Electronics Program supported by grants from Rockwell International Inc., Apple Computer Inc., GEC Ferranti Defence Systems Ltd., Italtel, General Electric Co., Day-Ray Products Inc., Southern California Edison, and MagneTek. prediction for the case where no compensating ramp is used.

A continuous-time model was proposed in [6], where effort is focused on improvement of frequency response prediction up to half the switching frequency. It reveals that peaking at half the switching frequency is possible in various transfer functions when the duty-ratio is close to 0.5 and no compensating ramp is used. Under this circumstance, low-frequency models are not sufficient. However, the proposed continuous-time form of currentloop gain is inconsistent with the corresponding closedloop transfer function.

In reviewing existing models, one realizes that none of them has been able to predict all the salient features associated with current programming. It is the purpose of this paper to present a unified model for current-programmed converters which incorporates all the salient features, including subharmonic oscillations, and to present an analog injection technique for measurement of current-loop gain. A "stability parameter" $Q_{s}$, related to the converter duty ratio and the compensating ramp, emerges as the central quantity of interest.

Definitions and notation adopted here are the same as in [4].

\section{Duty-Ratio Control Law}

A desired form of small-signal low-frequency model of a current-programmed converter is shown in Fig. 1. This is essentially the same as in Figs. 11-13 of [4], except that a general canonical model for the power stage has been incorporated. Values of the parameters of the canonical model can be found in [10]. The modulator gain $F_{m}$ and coefficients $\alpha k$ (replacing $G_{6}$ ) and $\beta k$ (replacing $H_{3}$ ) reflect, respectively, the influence that the error current $\hat{i}_{c}-\hat{i}_{l}$, line voltage $\hat{v}_{g}$, and output voltage $\hat{v}$ may have on the duty ratio $\hat{d}$.

From the geometry of the inductor current waveform in steady state (in solid lines in Fig. 2) and with slopes $m_{1}, m_{2}$, and $m_{c}$ constant, one can obtain two equations:

$$
\left\{\begin{array}{l}
i_{l}=i_{c}-m_{c} d T_{s}-\frac{1}{2} m_{1} d T_{s} \\
i_{l}=i_{c}-m_{c} d T_{s}-\frac{1}{2} m_{2} d^{\prime} T_{s} .
\end{array}\right.
$$




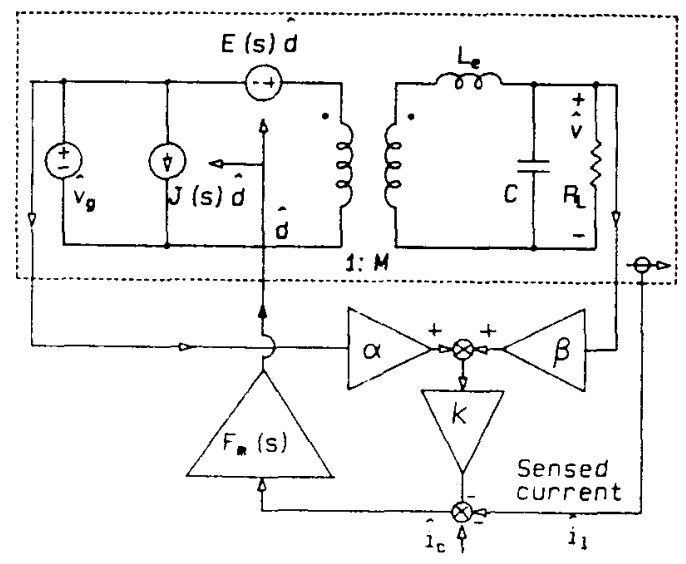

Figure 1: Small-signal low-frequency model of a currentprogrammed converter. It is shown later that the frequency response of the modulator gain $F_{m}(s)$ is a simple pole at $\omega_{p}=\left(\omega_{s} / 2\right) / Q_{s}$.
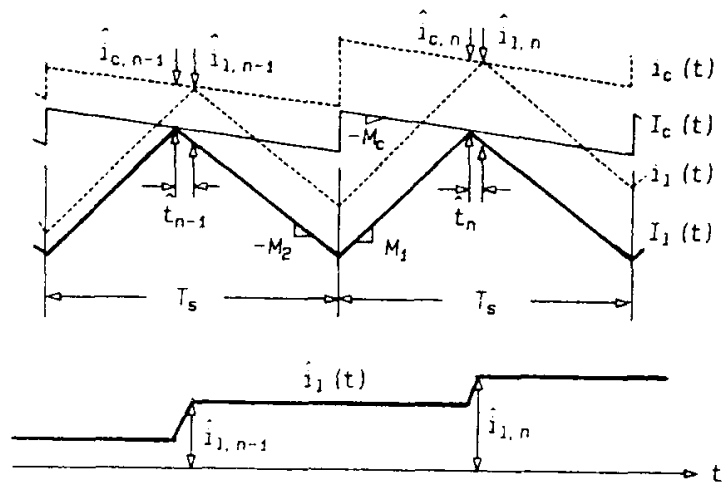

Figure 2: Geometries of inductor currents in steady and perturbed states.

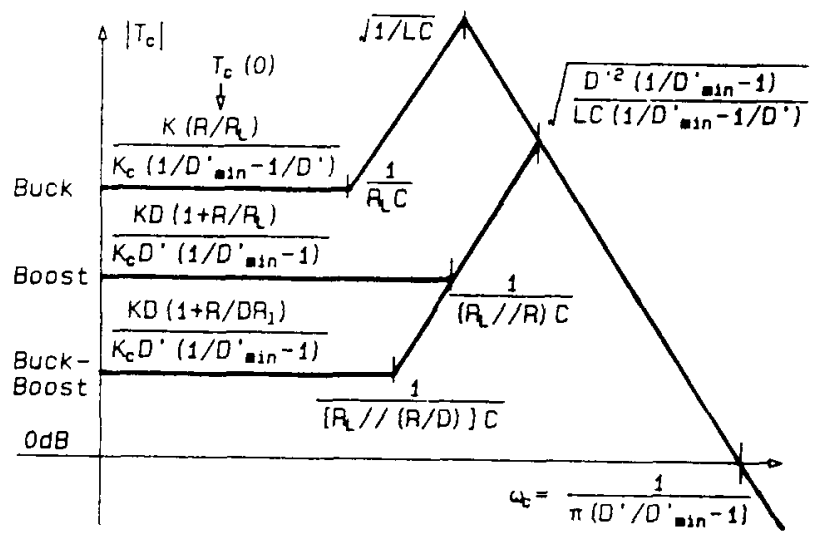

Figure 3: Current-loop gains for three basic converters.
These equations are written for steady state in order to be consistent with the canonical model for the power stage, which is derived by perturbation of a periodic steady-state solution to a linear time-varying system.

To satisfy two equations simultaneously, additional information is needed, which is found [12] in the following unified expressions for $m_{1}$ and $m_{2}$ :

$$
\left\{\begin{array}{l}
m_{1}=d^{\prime} v_{o f f} / L \\
m_{2}=d v_{o f f} / L
\end{array}\right.
$$

where $v_{o f f}$ is the sum of the two switch voltages [13]. Subscript "off" is used since this voltage is the voltage stress a switching device is subjected to when it is in the off state.

Substitution of Eq.(2) into either form of Eq.(1) leads to the same expression:

$$
i_{l}=i_{c}-m_{c} T_{s} d-\frac{d d^{\prime} T_{s}}{2 L} v_{o f f} .
$$

Perturbation of Eq.(3) yields

$$
\hat{i}_{l}=\hat{i}_{c}-T_{s}\left[M_{c}+\frac{1}{2}\left(M_{1}-M_{2}\right)\right] \hat{d}-\frac{D D^{\prime} T_{s}}{2 L} \hat{v}_{o f f}
$$

This equation can be further manipulated into the desired form of the duty-ratio control law,

$$
\hat{d}=\frac{K R\left(\hat{i}_{c}-\hat{i}_{l}\right)}{V_{o f f}\left(n D^{\prime}-D\right)}-\frac{D D^{\prime}}{V_{o f f}\left(n D^{\prime}-D\right)}\left(\alpha \hat{v}_{g}+\beta \hat{v}\right),
$$

where $K=2 L / R T_{s}, n=1+2 M_{c} / M_{1}$, and $\alpha=1,0$, and 1 , and $\beta=0,1$, and 1 , respectively, for the buck, boost, and buck-boost converters. Hence, it is found that, in Fig. 1,

$$
\begin{aligned}
F_{m} & =\frac{K R}{V_{o f f}\left(n D^{\prime}-D\right)}, \\
k & =\frac{D D^{\prime}}{K R} .
\end{aligned}
$$

The modulator gain $F_{m}$ is different from the result of [4] only in that $n D^{\prime}-D$ replaces $n D^{\prime}$, and is in agreement with that of [11]. It will be seen that $n D^{\prime}-D$ can be expressed in a different, more useful form. The dutyratio control law of $\mathrm{Eq} .(5)$ is a modification of that proposed in [4].

\section{Current-Loop Gain $T_{c}$}

\subsection{Determination of $D_{m i n}^{\prime}$}

By the same procedure as in [4], the duty-ratio control law of Eq.(5) leads to the current-loop gain $T_{c}(s)$. The results, shown in Fig 3 , are qualitatively the same as in [4]. The salient feature is that the final asymptote 
is the same for all three converters, crossing zero $\mathrm{dB}$ at $\omega_{c}=\omega_{s} / \pi\left(n D^{\prime}-D\right)$, which is quantitatively different from the result of [4] in that $n D^{\prime}-D$ replaces $n D^{\prime}$.

The quantity $n D^{\prime}-D$ is a function of the compensating ramp $M_{c}$ and the converter operating point represented by the duty ratio $D=1-D^{\prime}$. As $D$ is increased from zero, or the complementary duty ratio $D^{\prime}$ decreased from unity, $n D^{\prime}-D$ decreases and reaches zero before $D$ reaches unity. As already shown in [4], $n D^{\prime}-D$ must be greater than zero for stability of the current loop, so it is seen now that $\omega_{c}$ goes to infinity at the same stability limit.

It is therefore convenient to express $n D^{\prime}-D$ in terms of a new parameter $D_{\min }^{\prime}=1-D_{\max }$ which is the lowest value of $D^{\prime}$ for which stability is maintained. Straightforward algebra leads to.

$$
n D^{\prime}-D=\frac{D^{\prime}-D_{\min }^{\prime}}{D_{\min }^{\prime}}=\frac{D-D_{\max }}{1-D_{\max }}
$$

where

$$
D_{\min }^{\prime} \equiv \frac{0.5}{\left(1+M_{c} / M_{1}\right)} .
$$

It is seen that $D_{\min }^{\prime}=0.5$ if $M_{c}=0$, and decreases with increasing $M_{c}$, corresponding to the well-known result that increasing compensating ramp extends stability upwards from $D=0.5$.

Hence, a low-entropy expression [14] for $\omega_{c}$ is

$$
\omega_{c}=\frac{\omega_{s}}{\pi\left(D^{\prime} / D_{\min }^{\prime}-1\right)},
$$

which is in terms of the operating point parameter $D^{\prime}$ relative to its minimum value $D_{\min }^{\prime}$ for stability. Other salient features in Fig. 3 are also expressed in terms of $D_{\min }^{\prime}$. In addition, the zero-frequency value $T_{c}(0)$ of the current-loop gain is expressed in terms of $K / K_{c} \equiv$ $K / K_{\text {crit }}$ which, as described in [4], represents the ratio of the inductance $L$ to its minimum value to maintain continuous conduction.

Extension of $T_{c}(s)$ to include the sampling effect shows that $\omega_{c}$ is not necessarily the current loop crossover frequency because of the presence of an additional pole in $T_{c}(s)$.

\subsection{Sampling Effect: Additional Pole $\omega_{p}$, and determination of "Stability $\mathrm{Pa}$ - rameter" $Q$ s}

The approach is to find a rational function approximation of the sampled representation of the closed-loop transfer function $\hat{i}_{i} / \hat{i}_{c}$ of the current-programmed power stage, from which the presence of one additional pole in $T_{c}(s)$ is inferred.
From the geometry of the currents in Fig. 2, one can write two discrete-time equations:

$$
\left\{\begin{array}{l}
\Delta i_{c, n+1}=\Delta i_{l, n}+M_{1} \Delta t_{n+1}+M_{c} \Delta t_{n+1} \\
\Delta i_{l, n+1}=\Delta i_{c, n+1}-M_{c} \Delta t_{n+1}+M_{2} \Delta t_{n+1} .
\end{array}\right.
$$

Two results can be obtained from this set of equations: one is the duty-ratio modulator gain $F_{m}$, and the other is a discrete-time transfer function $\Delta i_{1} / \Delta i_{c}$.

Subtraction of the first expression from the second and then rearranging yields

$$
\hat{d}_{n+1} \equiv \frac{\Delta t_{n+1}}{T_{s}}=\frac{\Delta i_{c, n+1}-\left(\Delta i_{l, n+1}+\Delta i_{l, n}\right) / 2}{\left[M_{c}+\left(M_{1}-M_{2}\right) / 2\right] T_{s}}
$$

With the restriction of low-frequency perturbation, one has $\Delta i_{l, n+1} \approx \Delta i_{l, n}$, which in turn gives

$$
\hat{d}=\frac{\hat{i}_{c}-\hat{i}_{i}}{\left[M_{c}+\left(M_{1}-M_{2}\right) / 2\right] T_{s}}=\frac{K R\left(\hat{i}_{c}-\hat{i}_{i}\right)}{V_{o f f}\left(n D^{\prime}-D\right)},
$$

which shows the same coefficient as in Eq.(5), and verifies the result for the modulator gain $F_{m}$ derived above.

The discrete transfer function from control $\Delta i_{c}$ to inductor current $\Delta i_{l}$ is derived by introduction of the ztransform into the two equations in Eq.(11), which leads to

$$
\frac{\Delta i_{l}(z)}{\Delta i_{c}(z)}=a \frac{z}{z-1+a},
$$

where $a \equiv\left(M_{1}+M_{2}\right) /\left(M_{1}+M_{c}\right)=2 D_{\min }^{\prime} / D^{\prime}$.

To derive a continuous expression for the current transfer function, two more relationships are needed: 1) the connection between the $z$ domain and the sampled-Laplace domain; and 2) the connection between sampled- and continuous-Laplace domains. The first is the identity $z=e^{s T}$. The second is provided by the concept of "equivalent hold" proposed in [15], which states: In the small-signal limit, the continuous quantity $\hat{i}_{1}$ is related to its sampled counterpart by the transfer function of a zeroth-order-hold circuit.

Applying this property, one obtains

$$
\hat{i}_{l}(s)=a \frac{e^{s} T_{v}}{e^{s T_{r}}-1+a} \frac{1-e^{-s T_{v}}}{s} \hat{i}_{c}^{*}(s),
$$

where the asterisk represents a sampled quantity and

$$
\hat{i}_{c}^{*}(s)=\frac{1}{T_{s}} \sum_{n=-\infty}^{\infty} \hat{i}_{c}\left(s+\frac{j 2 n \pi}{T_{s}}\right) \approx \frac{1}{T_{s}} \hat{i}_{c}(s) .
$$

The approximation is made possible by the fact that the control current can be taken to be a pure sinusoid, and hence contributions of sidebands are negligible if a narrow-band analyzer is used for measurement. 


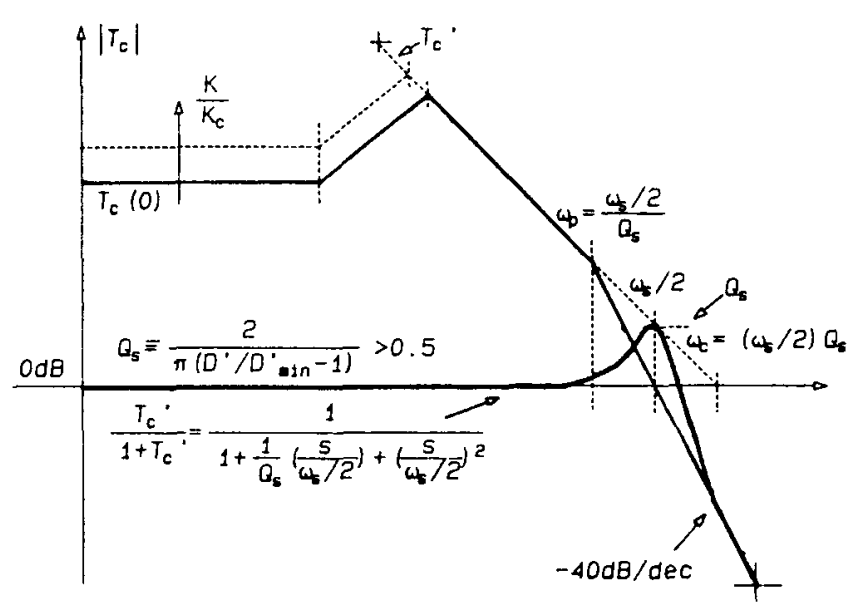

Figure 4: Current-loop gain for "stability parameter" $Q_{s}>0.5 . T_{c}$ crosses over at $\omega_{s} / 2$ on a double slope; closed-loop quadratic peaks up.

To simplify further the current transfer function, one can invoke the following modified Padé approximation for the complex exponential, used implicitly in [6]:

$$
e^{-s T_{s}}=\frac{1-\frac{1}{2 / \pi}\left(\frac{s}{\omega_{s} / 2}\right)+\left(\frac{s}{\omega_{s} / 2}\right)^{2}}{1+\frac{1}{2 / \pi}\left(\frac{s}{\omega_{s} / 2}\right)+\left(\frac{s}{\omega_{s} / 2}\right)^{2}} .
$$

This approximation enjoys good accuracy up to half the switching frequency. Substitution of Eq.(17) into Eq.(15) yields

$$
\frac{\hat{i}_{l}(s)}{\hat{i}_{c}(s)}=\frac{1}{1+\frac{1}{Q_{s}}\left(\frac{s}{\omega_{v} / 2}\right)+\left(\frac{s}{\omega_{s} / 2}\right)^{2}}
$$

where $Q_{s}=\frac{2}{\pi(2 / a-1)}$, or

$$
Q_{s} \equiv \frac{2}{\pi\left(D^{\prime} / D_{\min }^{\prime}-1\right)}
$$

It is seen that the current transfer function is a standard low-pass quadratic with its corner located at half the switching frequency, and whose $Q$-factor $Q_{s}$ can be effectively controlled by the compensating ramp $M_{c}$ through the parameter $D_{\min }^{\prime}$.

Moreover, the expression for $Q_{s}$ reveals explicitly that oscillation is possible if $Q$, goes to infinity, that is, if the inequality $D^{\prime}>D_{\min }^{\prime}$ is violated, which is exactly the conventional criterion for stability of the current loop $[3,4]$. Since this potential oscillation will be at half the switching frequency, it is commonly referred to as subharmonic oscillation.

The denominator of Eq.(18) is typical of the closedloop response of a system having a two-pole loop gain in

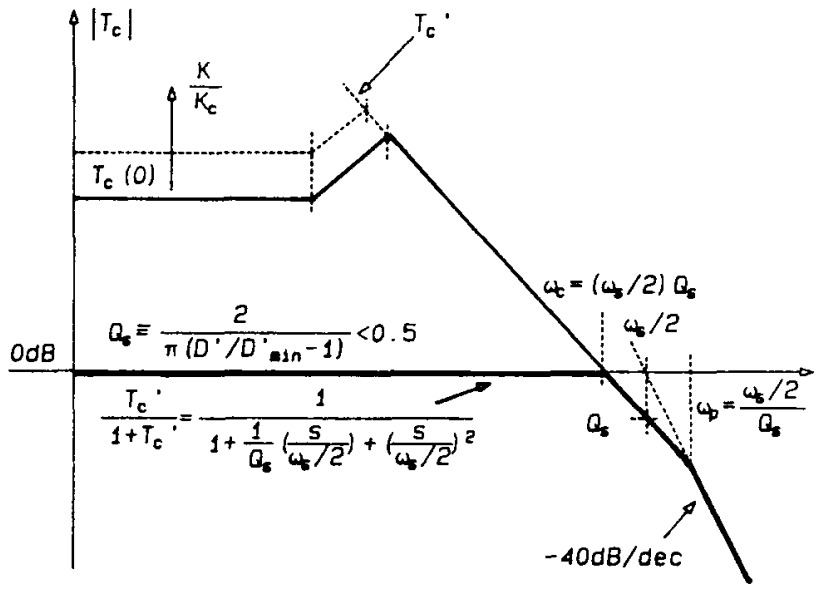

Figure 5: Current-loop gain for $Q_{s}<0.5 . T_{c}$ crosses over at $\omega_{c}$ on a single slope; closed-loop quadratic has real poles at $\omega_{c}$ and $\omega_{p}$.

the neighborhood of the crossover frequency. If in this neighborhood the loop gain is $T_{c}^{\prime}(s)$, then $T_{c}^{\prime}(s)$ can be inferred from

$$
\frac{T_{c}^{\prime}}{1+T_{c}^{\prime}}=\frac{1}{1+\frac{1}{Q_{s}}\left(\frac{s}{\omega_{s} / 2}\right)+\left(\frac{s}{\omega_{*} / 2}\right)^{2}},
$$

which leads to

$$
T_{c}^{\prime}(s)=\frac{1}{\frac{s}{\omega_{c}}\left(1+\frac{s}{\omega_{v}}\right)},
$$

where

$$
\begin{aligned}
& \omega_{p}=\frac{\left(\omega_{s} / 2\right)}{Q_{s}}=\frac{\pi \omega_{s}\left(D^{\prime} / D_{\min }^{\prime}-1\right)}{4} \\
& \omega_{c}=\left(\omega_{s} / 2\right) Q_{s}=\frac{\omega_{s}}{\pi\left(D^{\prime} / D_{\min }^{\prime}-1\right)}
\end{aligned}
$$

It is seen that $\omega_{c}$ is identical to that shown in Fig. 3 which identifies the high-frequency asymptote of $T_{c}(s)$, and hence the sampling effect can be considered responsible for introducing an additional pole $\omega_{p}$ in $T_{c}(s)$.

It can therefore be concluded that the current-loop gains $T_{c}$ of Fig. 3 may be augmented by the additional pole $\omega_{p}$ to account for the sampling effect. Two cases of interest, for two values of $Q_{s}$, are shown in Figs. 4 and 5 . In each, appropriate expressions for $T_{c}(0)$ and adjacent corner frequencies can be taken from Fig. 3 for the different converters. Increasing $K / K_{c}$ (larger inductance) increases only the low-frequency range of $T_{c}(s)$.

In Fig. 4 for $Q_{s}>0.5, T_{c}$ crosses over at $\omega_{s} / 2$ on a double slope and the resulting closed-loop response, represented by $T_{c}^{\prime} /\left(1+T_{c}^{\prime}\right)$, has a corresponding peak at $\omega_{s} / 2$. 
In Fig. 5, for $Q_{s}<0.5, T_{c}$ crosses over at $\omega_{c}$ less than $\omega_{s} / 2$ on a single slope, and the additional pole $\omega_{p}$ is beyond crossover. The resulting closed-loop response has two real poles $\omega_{c}$ and $\omega_{p}$.

A wealth of useful design-oriented information is available from these low-entropy results. First, the final $-40 d B / d e c$ asymptote for $T_{c}$ is fixed in position, crossing $0 \mathrm{~dB}$ at $\omega_{s} / 2$. Whether current-loop gain $T_{c}$ crossover occurs at or below $\omega_{s} / 2$ depends solely on $Q_{s}$, which is a function of the operating point $D^{\prime}$ relative to $D_{\min }^{\prime}$ given by Eq.(19). In turn, $D_{\min }^{\prime}$ is determined by the slope of the compensating ramp $M_{c}$, by Eq.(9).

Thus, if all quantities are considered constant except $M_{c}$, increasing $M_{c}$ results in a smaller $D_{\min }^{\prime}$ and a smaller $Q_{s}$, lowering $\omega_{c}$ and changing the configuration of $T_{c}$ from that of Fig. 4 towards that of Fig. 5. Alternatively, if all quantities are constant except the operating point parameter $D^{\prime}$, increasing $D^{\prime}$ (lower $D$ ) also results in a smaller $Q_{s}$ with the same consequences.

Since Figs. 4 and 5 illustrate the familiar loopgain/closed-loop relationships for a single-loop system, in which increasing loop-gain slope at crossover(lower phase margin) leads to peaking in the closed-loop response, it emerges that $Q_{3}$ is the central quantity of interest in the current-loop gain, and could be referred to as a "stability parameter," since $Q$, approaches infinity in the stability limit of $D^{\prime}$ declining to $D_{\min }^{\prime}$. Further, a smaller $Q$, inexorably results in a lower $T_{c}(0)$.

Finally, a unified model for current-programmed converters with the sampling effect accounted for is obtained by expression of the modulator gain $F_{m}(s)$ in Fig. 1 as $F_{m} /\left(1+s / \omega_{p}\right)$. Various closed-loop transfer functions of interest can be derived from this model, and the low-pass quadratic of Eq.(18), in terms of the stability parameter $Q_{s}$, appears in all of them.

\section{Loop Gain Measurement}

Figure 6 illustrates one particular way of representing a digital injection measurement. A digital injection technique injects a perturbation of duty ratio $\hat{d}_{z}^{*}$ at the output terminal of the duty ratio modulator [16]. To represent its discrete nature, a sampler, shown ahead of the modulator, operates in a quasi-periodic fashion which normally complicates modeling. This quasiperiodicity is characterized in [15], where it is shown that in the small-signal limit, the quasi-periodicity is inconsequential. In other words, the sampler can be treated as a periodic device.

From the simple model in Fig. 6, one can write:

$$
\hat{d}_{y}^{*}=\left[-\hat{d}_{x}^{*} G_{d i}(s)\right]^{*} F_{m}=-\left[G_{d i}(s) F_{m}\right]^{*} \hat{d}_{x}^{*}
$$

where $G_{d i}(s)$ denotes the duty-ratio-to-inductor-current transfer function, and $F_{m}$ denotes the modulator gain

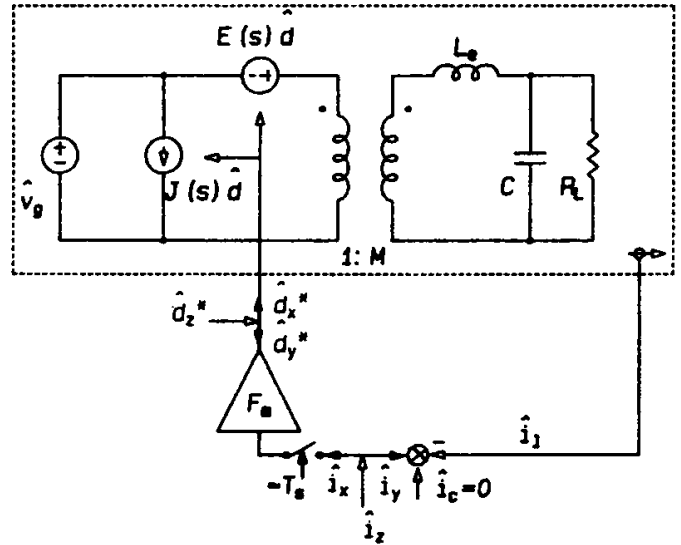

Figure 6: Measurement of current-loop gain using digital injection $\hat{d}_{z}^{*}$ or analog injection $\hat{i}_{z}$.

which has been taken to be a real number, although the actual values of these quantities are of no concern for this discussion. The above equation gives

$$
-\frac{\hat{d}_{y}^{*}}{\hat{d}_{x}^{*}}=\left[G_{d i}(s) F_{m}\right]^{*}=T_{c}^{*}(s)
$$

Therefore, the digital injection technique actually measures a sampled version of loop gain. A digital injection is necessary if transistor current is sensed, since the sensed current is both pulse-width and amplitude modulated and a narrow-band analyzer cannot distinguish one from the other. On the other hand, the digital injection technique loses information close to and above half the switching frequency because of the effect of frequency folding associated with sampling.

Since current-loop gain crossover frequency can be as high as half the switching frequency, for a complete characterization a measurement technique is needed which can retain information up to and above half the switching frequency.

In a two-switch converter, the transistor current is identical to the rising portion of the inductor current, and sensing an inductor current is equivalent to sensing a transistor current. This equivalence brings a major benefit, that is, the sensed current is nonpulsating, which in turn suggests that the conventional analog injection technique for measurement of loop gain can be used, as also illustrated in Fig. 6 by an injected analog current $\hat{i}_{z}$.

\section{Experimental Verifications}

To verify theoretical predictions from the unified model and to check the proposed measurement technique, a 


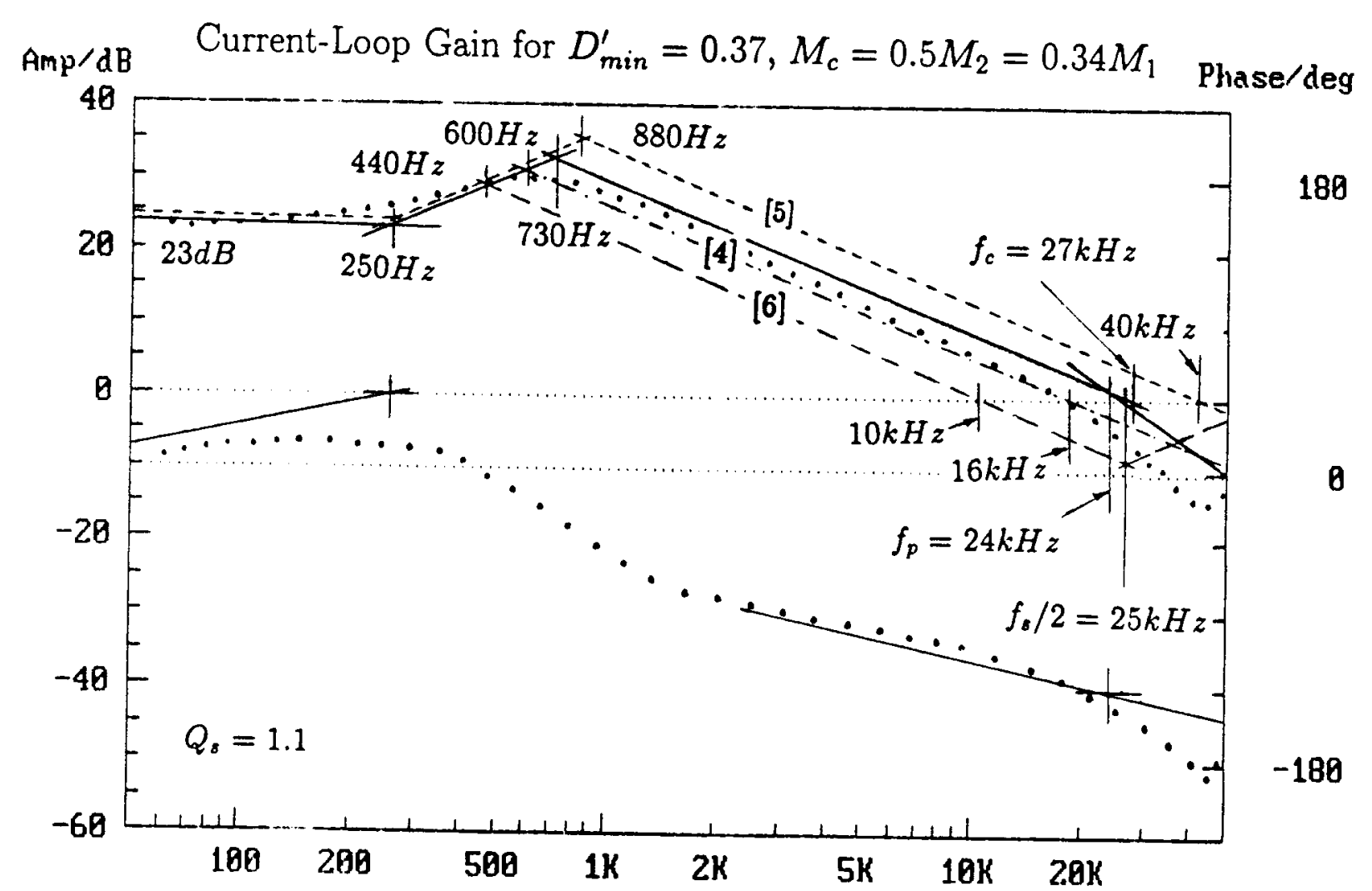

Figure 9: Measured results(data points) and predicted asymptotes of current-loop gain for $D_{\min }^{\prime}=0.37$. Solid asymptotes: unified model; others: according to indicated Reference numbers.

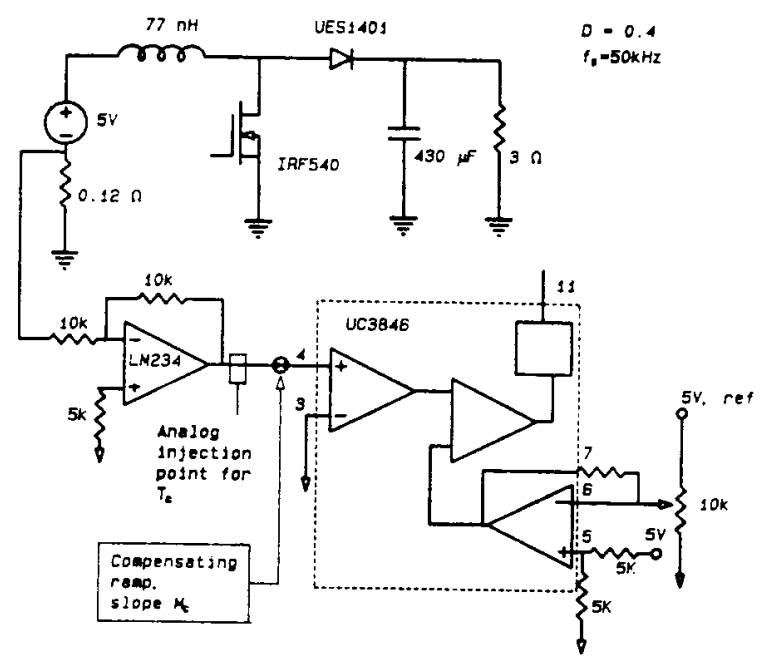

Figure 7: Test boost converter.

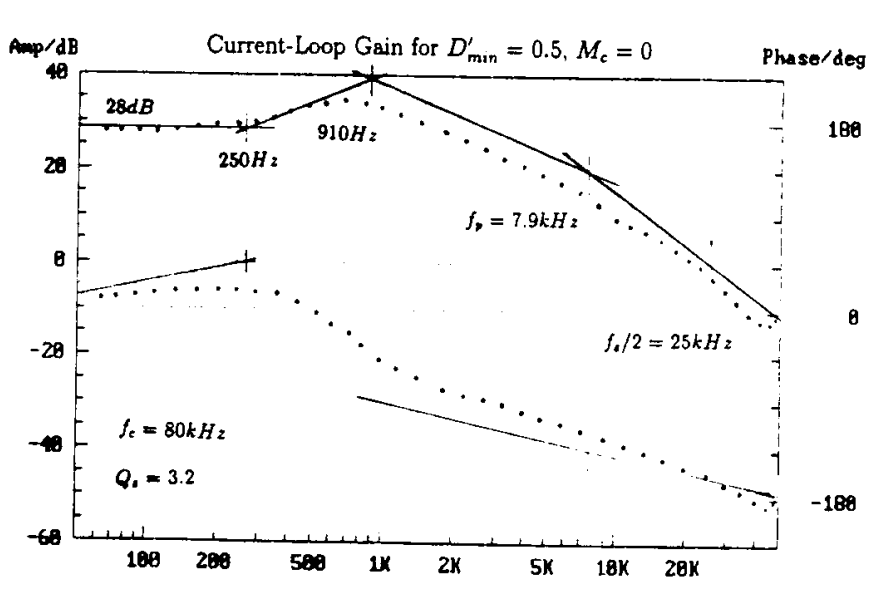

Figure 8: Measured results(data points) and prediction (in asymptotes) of current-loop gain for $D_{\min }^{\prime}=0.5$. Existence of $\omega_{p}$ is clearly seen. 
prototype boost converter has been constructed and extensive measurements performed.

Figure 7 shows a test boost converter switching at $50 \mathrm{kHz}$. The nonpulsating inductor current is sensed, and the input voltage source is floated to permit the current sense resistor to be connected to power ground. The LM234 is introduced to reduce noise in the sensed current waveform and to allow convenient analog voltage injection at its output for measurement of currentloop gain $T_{c}$.

Various values of compensating ramp slope $M_{c}$ are used to establish corresponding values of $D_{\min }^{\prime}$ and stability parameter $Q_{8}$. All measurements are made at a converter operating point $D=0.4, D^{\prime}=0.6$ which allows both a margin for modulation and a large value of $Q_{s}$ so that possible peaking at $f_{\mathrm{s}} / 2$ is exposed.

Figure 8 shows the predicted asymptotes and measured results of current-loop gain for $D_{\min }^{\prime}=0.5$ (no compensating ramp, $M_{c}=0$ ). Since the operating point is $D^{\prime}=0.6$, little larger than $D_{\min }^{\prime}, Q_{s}$ is high: from Eq.(19) $Q_{s}=3.2>1$, from Eq.(22) the additional pole is at $f_{p}=7.9 \mathrm{kHz}$, and the unified model predicts that $T_{c}$ crosses over at $f_{s} / 2=25 \mathrm{kHz}$ on a double slope. Agreement between prediction and measurement is good, and the presence of the additional pole $\omega_{p}$ can clearly be seen, especially from the phase measurement.

Figure 9 shows the same comparison for $D_{\min }^{\prime}=0.37$ $\left(M_{c}=M_{2} / 2\right)$, together with additional magnitude asymptotes as predicted from models in $[4,5,6]$, respectively. Since in the unified model $Q_{s}=1.1>1$, $f_{p}=25 / 1.1=24 k \mathrm{kz}$ and $f_{c}=25 \times 1.1=27 \mathrm{kHz}$ are very close to $f_{z}=25 \mathrm{kHz}$, in good agreement with measurement. On the other hand, $f_{c}$ is predicted respectively at $16 \mathrm{kHz}, 40 \mathrm{kHz}$, and $10 \mathrm{kHz}$ by the models in $[4,5,6]$. Because of the fact that the corner $\omega_{p}$ is very close to the crossover frequency $(25 k \mathrm{~Hz})$, the measured result crosses over at less than $20 \mathrm{kHz}$. It can therefore be concluded that predictions from $[4,6]$ tend to be lower, while the prediction from [5] tends to be higher than the actual value. The existence of $\omega_{p}$ is also seen in the amplitude and phase measurements.

Figure 10 shows again good agreement between the unified model prediction and the measured results of current-loop gain for $D_{\min }^{\prime}=0.3\left(M_{c}=M_{2}\right)$. Since $Q_{3}=0.64$ is now less than unity, $T_{c}$ crosses over on a single slope near $f_{c}=0.64 \times 25=16 k \mathrm{~Hz}$, showing that improved stability is obtained by increasing the compensating ramp to give a lower $Q_{\text {s. }}$.

Figure 11 shows the measured results for the controlto-current transfer function of Eq.(18) for $D_{\min }^{\prime}=0.5$ $\left(M_{c}=0\right)$. As predicted, for the high $Q$, of 3.2 , peaking at $f_{s} / 2=25 \mathrm{kHz}$ is present, as in Fig. 12 for the controlto-output transfer function which also contains the same low-pass quadratic as in Eq.(18).

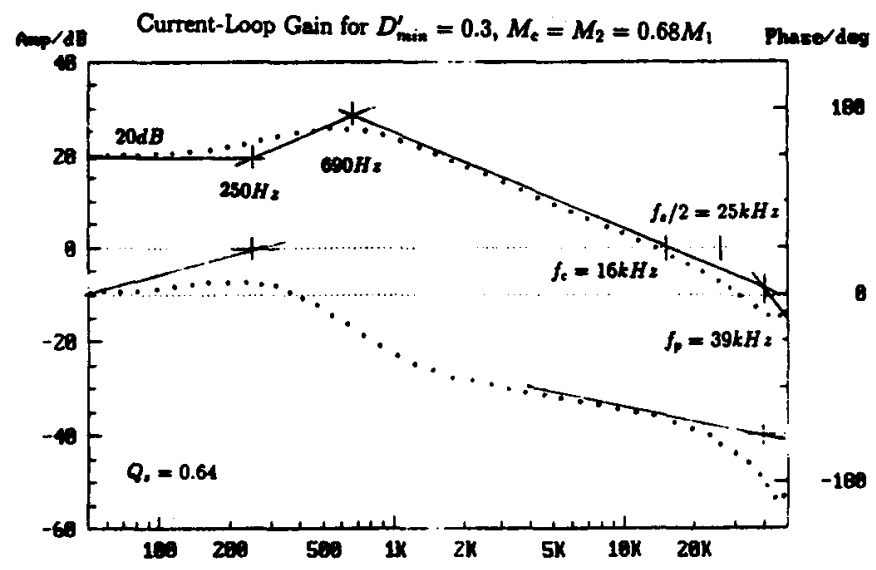

Figure 10: Measured results(data points) and predicted asymptotes of current-loop gain for $D_{\min }^{\prime}=0.3$. The additional pole $\omega_{p}$ lies above $\omega_{s} / 2$.

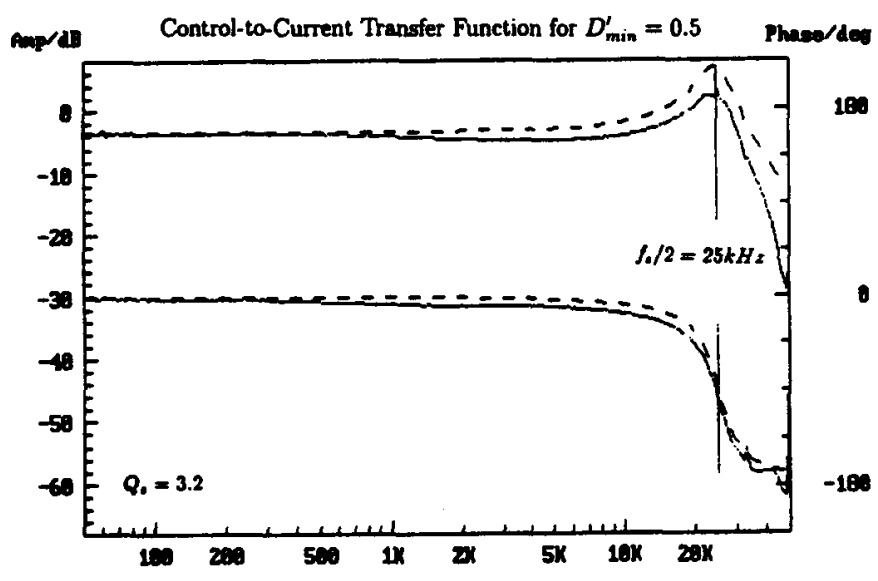

Figure 11: Measured(solid lines) and predicted(dashed lines) control-to-inductor-current transfer function.

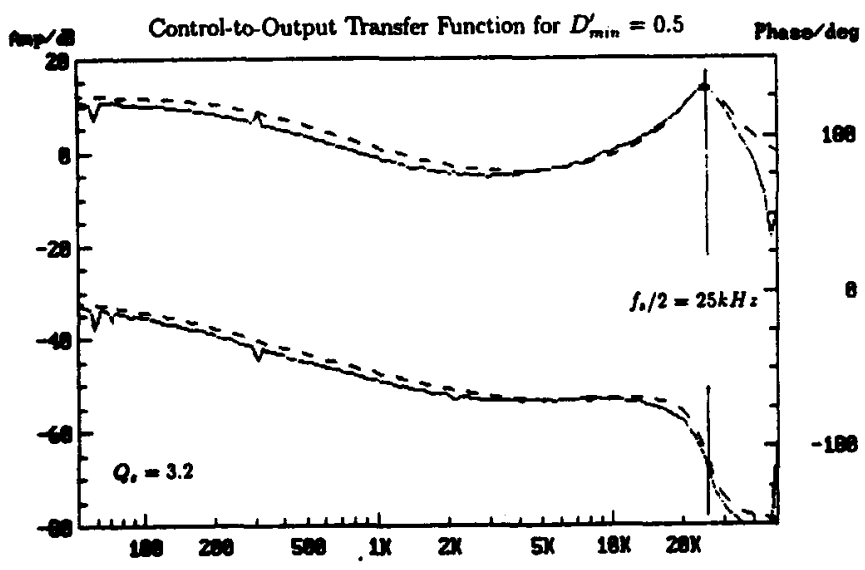

Figure 12: Measured(solid lines) and predicted(dashed lines) control-to-output-voltage transfer function. 


\section{Conclusions}

A unified model for a current-programmed converter is established as in Fig. 1. Although the format is the same as in [4], a modified duty-ratio control law leads to correspondingly modified expressions for $F_{m}$ and $k$ as given in Eqs.(6) and (7), and also for the loop gain $T_{\mathrm{c}}$ shown in Fig. 3 .

It is well-known that use of a compensating ramp of slope $M_{c}$ extends upwards from 0.5 the limit of the duty ratio $D$ for which stability is maintained. It is found convenient to introduce a new parameter $D_{\min }^{\prime}$, defined by $M_{c}$ as in Eq. 9, which represents the minimum value of the complementary duty ratio $D^{\prime}=1-D$ for which stability is maintained.

It is also well-known that the instability is caused by the sampling effect, which is not accounted for in the loop gain shown in Fig.3. The sampling effect is here accounted for by an extension of the previous model from which the presence of an additional pole $\omega_{p}$ in $T_{c}$, given by Eq.(22), is inferred and incorporated in the modulator gain $F_{m}(s)$ in Fig. 1.

The presence of the additional pole $\omega_{p}$ causes the final high-frequency asymptote of $T_{c}$ to be $-40 \mathrm{~dB} / \mathrm{dec}$, as shown in Figs. 4 and 5. A salient feature is that this final asymptote is fixed in position, crossing zero $d B$ at $\omega_{s} / 2$, and that consequently the loop-gain crossover frequency cannot exceed half the switching frequency regardless of any component values or converter operating point. Whether crossover occurs at or below $\omega_{s} / 2$ is determined by the value of the operating point $D^{\prime}$ relative to $D_{\min }^{\prime}$ through another new parameter $Q_{s}$ defined in Eq.(19). The "stability parameter" $Q$ s emerges as the central quantity of interest in the current-loop gain $T_{c}$, since its value determines not only the crossover frequency but also the degree of peaking, which always occurs at $\omega_{s} / 2$, in the low-pass quadratic of Eq.(18) that is contained in all of the closed-loop transfer functions.

The unified model is verified by experimental measurements of current-loop gain on a test boost converter. An analog signal injection technique is enabled by sensing the (nonpulsating) inductor current instead of the pulsating switch current, which does not change the nature of the current loop. Figure 8 shows $T_{c}$ for $Q_{s}=3.2$, a high value since $D^{\prime}=0.6$ is little larger than $D_{\min }^{\prime}=0.5$; the presence of the additional pole $\omega_{p}$ at $7.9 \mathrm{kHz}$, well below crossover, is clearly visible. The resulting closed-loop control-to-current and control-tooutput transfer functions of Figs.11 and 12 show the expected peaking.

\section{References}

[1] C. W. Deisch, "Simple Switching Control Method Changes Power Converter into a Current Source," IEEE PESC Record, 1978, pp. 300-306.
[2] A. Capel, G. Ferrante, D. O'Sullivan, and A. Weinberg, "Application of the Injected Current Model for the Dynamics of Switching Regulators with the New Concept of LC3 Modulator," IEEE PESC Record, 1978, pp. 135147.

[3] S.-P. Hsu, A. Brown, L. Rensink, and R. D. Middlebrook, "Modeling and Analysis of Switching DC-to-DC Converters in Constant-Frequency Current-Programmed Mode," IEEE PESC Record, pp. 284-301.

[4] R. D. Middlebrook, "Topics in Multiple-Loop Regulators and Current-Mode Programming," IEEE PESC Record, 1985, pp. 716-732.

[5] G. C. Verghese, C. A. Bruzos, and K. N. Mahabir, "Averaged and Sampled-Data Models for Current Mode Control: a Reexamination," IEEE PESC Record, 1989, pp. 484-491.

[6] R. B. Ridley, "A New Continuous-Time Model for Current-Mode Control," PCIM Conference Record, 1989, pp. 16-20.

[7] V. Vorpérian, "Analysis of Current-Mode Controlled PWM Converters Using the Model of the CurrentControlled PWM Switch," PCIM Conference Record, 1990.

[8] R. M. Tedder, "An Alternate Analysis of the CurrentProgrammed Model Leads to Simplified Input Filter Design Criteria," IEEE APEC Record, 1992, pp. 756-673.

[9] R. Redl, "High-Frequency Extension of the SmallSignal Model of the Constant-Frequency Current-ModeControlled Converters," IEEE PESC Record, 1991, pp. 466-472.

[10] R. D. Middlebrook, and S. M. Ćuk, "A General Unified Approach to Modeling Switching Converter Power Stages," IEEE PESC Record, 1976, pp. 18-34.

[11] F. C. Lee, Y. Yu, and M. F. Mahmoud, "A Unified Analysis and Design Procedure for a Standardized Control Module for Dc-to-Dc Switching Regulators," IEEE PESC Record, 1980, pp. 284-301.

[12] D. Maksimović, and S. M. Ćuk, "A Unified analysis of PWM Converters in Discontinuous Modes," International Power Conversion and Intelligent Motion Conference Record, June, 1989.

[13] S. Freeland and R. D. Middlebrook, "A Unified Analysis of Converters with Resonant Switches," IEEE PESC Record, 1987, pp. 20-30.

[14] R. D. Middlebrook, "Low-Entropy Expressions: the Key to Design-Oriented Analysis," Proc. IEEE Frontiers in Education, Twenty-First Annual Conference, Purdue University, 1991, pp. 399-403.

[15] B. Y. B. Lau and R. D. Middlebrook, "Small-Signal Frequency Response Theory for Piecewise-Constant TwoSwitched-Network Dc-to-Dc Converters," IEEE PESC Recond, 1986, pp. 186-200.

[16] B. H. Cho and F. C. Lee, "Measurement of Loop-Gain with the Digital Modulator," IEEE PESC Record, 1984, pp. 363-373. 